\title{
INVASÃO BIOLÓGICA POR Hovenia dulcis THUNB. EM FRAGMENTOS FLORESTAIS NA REGIÃO DO ALTO URUGUAI, BRASIL ${ }^{1}$
}

Luciane Cristina Lazzarin ${ }^{2}$, Ana Carolina da Silva ${ }^{3}$, Pedro Higuchi $^{3}$, Karine Souza $^{2}$, Jucelei Edson Perin $^{4}$ e Aline Pereira Cruz ${ }^{2}$

\begin{abstract}
RESUMO - Buscou-se caracterizar a composição florístico-estrutural do componente arbóreo de uma área contaminada por Hovenia dulcis Thunb., determinar a relação da distribuição da espécie invasora com o estágio sucessional e verificar a relação da presença da espécie invasora com padrões de diversidade e de estruturação da comunidade. $\mathrm{O}$ estudo foi realizado em uma área ciliar do reservatório da Usina Hidrelétrica de Machadinho, em Santa Catarina. O levantamento foi conduzido em 48 parcelas de $200 \mathrm{~m}^{2}$ cada, dispostas em oito blocos. Dentro das parcelas, todos os indivíduos arbóreos com Circunferência Medida à Altura do Peito (CAP) igual ou superior a $15,7 \mathrm{~cm}$ foram medidos (CAP) e identificados. Os dados foram analisados por meio dos descritores fitossociológicos, Índice de Invasão Biológica (IIB), índices de diversidade, escalonamento multidimensional não métrico, análise de variância multivariada permutacional, análise de espécies indicadoras e o índice de c-score. Foram amostradas 66 espécies, com a de maior Valor de Importância (VI) Ocotea puberula (Rich.) Nees (10,79\%). H. dulcis ocupou a sétima posição em VI (4,04\%), e o IIB foi de 0,47 . Os blocos foram classificados em grupos sucessionais médios e avançados, com diferenças florístico-estruturais entre eles $(p=0,001)$. $H$. dulcis associou-se com áreas em estágio sucessional mais inicial, com menor diversidade de espécies arbóreas e ausência de estruturação da comunidade, referente à segregação das espécies nas parcelas. Conclui-se que a invasão por $H$. dulcis não ocorreu de forma espacialmente homogênea, sendo determinada pelas características ecológicas da comunidade residente.
\end{abstract}

Palavras-chave: Espécies invasoras; Comunidade residente; Floresta Atlântica.

\section{BIOLOGICAL INVASION BY Hovenia dulcis THUNB. IN FOREST FRAGMENTS IN UPPER-URUGUAY REGION, BRAZIL}

\begin{abstract}
We aimed to characterize the floristic and structural composition of the tree component of an area contaminated by Hovenia dulcis Thunb., to determine the relationship of the distribution of the invasive species to the successional stage, and to verify the relationship between the presence of invasive species with patterns of diversity and community structure. The study was conducted in a riparian area of the water reservoir of Machadinho Hydreletric power plant, in SC state. The survey was conducted in 48 plots of $200 \mathrm{~m}^{2}$ each, arranged in eight block. Within plots, all trees with circumference at breast height (cbh) greater or equal to $15.7 \mathrm{~cm}$ were measured (cbh) and identified. The data were analyzed through the phytosociological descriptors, the biological invasion index (BII), diversity index, non-metric multidimensional scalling, permutational multivariate analysis of variance, analysis of indicator species, and the c-score index. A total of 66 species was sampled, with the highest relative importance value (IV) for Ocotea puberula (Rich.) Nees (10.79\%). H. dulcis ranked in seventh position of IV (4.04\%) and the BII was 0.47 . The blocks were classified into
\end{abstract}

\footnotetext{
${ }^{1}$ Recebido em 09.02.2015 aceito para publicação em 06.11.2015.

${ }^{2}$ Universidade do Estado de Santa Cararina, Programa de Pós-Graduação em Engenharia Florestal, Lages, Santa Catarina - Brasil.E-mail:<engeflora.piratuba@hotmail.com>,<karisouza@hotmail.com>e <a.line_cruz@yahoo.com.br>.

${ }^{3}$ Universidade do Estado de Santa Catarina, Centro Agroveterinário, Departamento de Engenharia Florestal, Lages, Santa Caratina - Brasil.E-mail: <carol_sil4@yahoo.com.br>e <higuchip@gmail.com>.

${ }^{4}$ Centro de Desenvolvimento Ambiental, Itá, Santa Catarina - Brasil. E-mail: <jusselei.perin@hotmail.com>.
} 
intermediate and advanced successional groups, with floristic and structural differences $(p=0.001)$ between them. $\boldsymbol{H}$. dulcis was associated with areas with an earlier successional stage, with lower diversity and a community with no structure, in relation to segregation of species within plots. We conclude that the invasion by H. dulcis did not occur homogeneously in the area, being influenced by ecological characteristics of the resident community.

Keywords: Invasive species; Resident community; Atlantic Forest.

\section{INTRODUÇÃO}

O sinergismo entre a fragmentação de hábitats e a invasão biológica por espécies exóticas é considerado um dos principais problemas ambientais da atualidade, pois tem causado a perda de biodiversidade. A invasão é ocasionada por meio da introdução de espécies alóctones, que aumentou a partir do século XVI, com as grandes navegações, e cresce atualmente em decorrência do comércio mundial e dos transportes marítimo, terrestre e aéreo (PERRINGS et al., 2002; ZILLER; ZALBA, 2007). A invasão biológica, além de ser um problema ambiental, também representa importante problema econômico, causando danos que têm custado centenas de bilhões de dólares no mundo (PIMENTEL et al., 2001).

No Brasil, entre as regiões onde o processo de fragmentação e invasão biológica é evidente, destacase a região do Alto Uruguai, onde há uma área ecotonal entre a Floresta Estacional Decidual (FED) e Floresta Ombrófila Mista (FOM). Nessa região, que passou por um histórico recente de colonização humana e desmatamento (GASPER et al., 2013a), a espécie arbórea invasora Hovenia dulcis Thunb., de origem asiática, ocorre de forma abundante em remanescentes florestais (MEYER et al., 2012). A elevada representatividade dessa em remanescentes florestais indica que ela se encontra bem adaptada às condições edafoclimáticas da região, de forma que seu caráter invasor pode estar relacionado às suas características ecológicas, como a presença de propágulos atrativos à fauna e rápido crescimento (LORENZI et al., 2003), além de potencial alelopático (WANDSCHEER et al., 2011). H. dulcis, conhecida como uva-do-japão, apresenta regeneração por sementes, sendo seu pedicelo (um pseudofruto) consumido por animais (LORENZI et al., 2003), fazendo que as sementes presentes na sua extremidade sejam dispersas facilmente.

Alguns questionamentos a respeito do processo de invasão biológica, fundamentais para a definição de estratégias de controle, referem-se ao entendimento sobre os diferentes níveis de invasibilidade do ambiente e ao impacto da espécie invasora sobre padrões de diversidade e organização da comunidade. Segundo Davis et al. (2005), a invasibilidade por espécies exóticas pode ser definida como "a suscetibilidade de um ambiente à colonização e ao estabelecimento por indivíduos de espécies que não pertencem à comunidade residente". Considerando que a fragilidade do ambiente à colonização por espécies exóticas pode variar ao longo do tempo e de acordo com a disponibilidade de recursos (DAVIS et al., 2000), pode-se supor que a invasibilidade de uma floresta apresenta variações de acordo com o estágio sucessional da floresta e com as exigências ecológicas das invasoras que ocorrem no entorno.

Diante do exposto, este trabalho teve como objetivo contextualizar a presença de $H$. dulcis em relação à sua comunidade residente em uma área ecotonal, entre FED e FOM, na região do Alto Uruguai. Buscou-se: i) caracterizar a composição florístico-estrutural do componente arbóreo de uma área contaminada por $H$. dulcis; ii) verificar a relação da distribuição da espécie invasora com o estágio sucessional da floresta; e iii) constatar a relação da presença da espécie invasora com padrões de diversidade e de estruturação da comunidade.

\section{MATERIAL E MÉTODOS}

A área de estudo está localizada em uma área ecotonal entre FED e FOM (classificação do IBGE, 2012), na área ciliar do reservatório da Usina Hidrelétrica de Machadinho, no Rio Uruguai, Município de Piratuba, SC.Aaltitude é de $518 \mathrm{~m}$ e as coordenadas, de $27^{\circ} 28^{\prime} 06,92^{\prime \prime}$ Sul e $51^{\circ} 45^{\prime \prime} 55,73$ " Oeste. O clima da região é classificado, segundo Köppen, como Cfa, que indica clima subtropical úmido, com temperatura média de $18,4^{\circ} \mathrm{C}$ e pluviosidade média de $1.650 \mathrm{~mm} /$ ano. Foram amostrados trechos de florestas ciliares com diferentes históricos de perturbação. Alguns trechos da área são de floresta natural, porém receberam corte seletivo no passado até o ano 1998. Outros trechos são áreas que foram 
suprimidas para pastagens ou lavouras, mas que se regeneraram naturalmente e receberam o plantio de mudas nativas da região para a recuperação.

O levantamento da composição florística e estrutural foi realizado em 48 parcelas de $200 \mathrm{~m}^{2}(10 \times 20 \mathrm{~m})$ cada, dispostas em oito blocos alocados de forma aleatória na área, sendo cada bloco composto de seis parcelas (Figura 1). Cada bloco teve a distância mínima de 100 m entre si. Os blocos 1, 2, 3 e 4 foram localizados em áreas onde, antes do alagamento pela usina hidrelétrica, eram utilizadas para o cultivo de pastagem ou para lavoura. Essas áreas ciliares foram cercadas em 2002 e receberam o plantio das mudas nativas em 2010. Assim, o que se observou no ano dessa avaliação (2014) foi um estrato arbóreo composto por árvores provenientes da regeneração natural, o que acontece desde 2002 (componente amostrado neste estudo) e um estrato de sub-bosque proveniente também da regeneração natural, mas também das mudas plantadas em 2010. Os blocos 5, 6, 7 e 8 estão localizados em áreas que não sofreram corte raso, somente cortes seletivos e, por isso, não receberam o plantio de mudas, sendo somente realizado o isolamento da área.

Em cada parcela, todos os indivíduos arbóreos com circunferência medida à altura do peito (CAP) igual ou superior a $15,7 \mathrm{~cm}$ foram medidos (CAP), identificados e tiveram a altura estimada. As espécies foram identificadas por meio da literatura (SOBRAL et al., 2013) e de especialistas. As famílias de angiospermas foram organizadas de acordo com o sistema APG III (ANGIOSPERM PHYLOGENY GROUP, 2009).

Foram calculados, para a comunidade, o índice de Shannon e a equabilidade de Pielou. A fim de determinar a suficiência amostral para o conhecimento da riqueza de espécies, foi elaborada uma curva de acumulação de espécies. Essa curva foi construída por meio do método de aleatorização, com 1.000 permutações, no programa estatístico R (R DEVELOPMENT CORE TEAM, 2014), junto com o pacote Vegan (OKSANEN et al., 2014). A distribuição dos valores de riqueza estimados para as unidades amostrais, por meio das permutações, foi inserida na curva, utilizando gráficos do tipo "boxplot”. Também, foram calculados os descritores fitossociológicos clássicos propostos por Mueller-Dombois e Ellenberg (2002): densidades, frequências, dominâncias e Valor de Importância (VI).

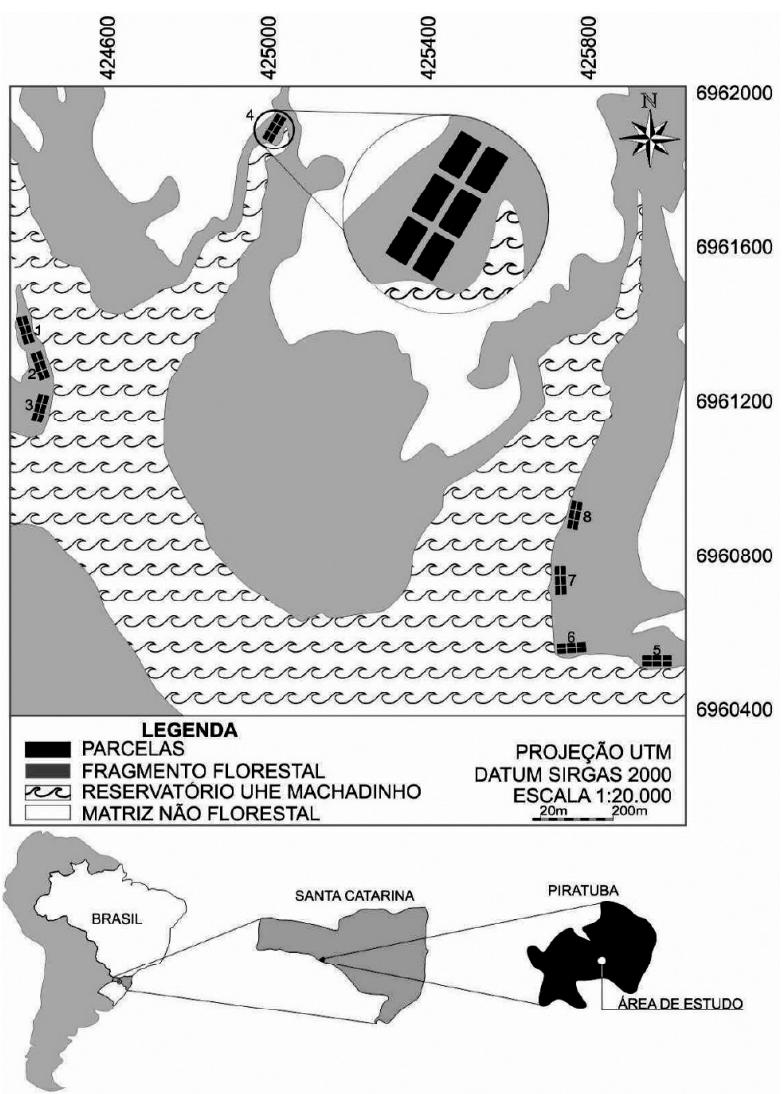

Figura 1-Blocos e parcelas estudados (ampliação para indicar o detalhe da distribuição das parcelas em cada bloco) em uma área de transição entre Floresta Estacional Decidual e Floresta Ombrófila Mista em Piratuba, SC

Figure 1-Blocks and plots studied (magnification to indicate the details of the distribution of plots in each block) in a transition area between Deciduous Forest and Araucaria Forest in the municipality of Piratuba, SC.

O nível de invasão biológica foi quantificado por meio do Índice de Invasão Biológica (IIB) proposto por Guidini et al. (2014): $I I B=\frac{\Sigma V I}{V I_{\max }}$, em que $\Sigma V I$ é o somatório do(s) valor(es) do(s) VI(s) da(s) espécie(s) exótica(s); VImax é o VI da espécie nativa de maior valor encontrado. Segundo Guidini et al. (2014), se IIB $\geq 1$, o grupo das espécies invasoras possui a maior importância relativa; se $1>$ IIB $\geq 0,5$, as invasoras possuem importância relativa inferior à da espécie nativa de maior VI, porém com valor superior à metade do VI da espécie nativa mais importante; e, se IIB $<0,5$, o grupo das invasoras possui importância relativa inferior

Revista Árvore, Viçosa-MG, v.39, n.6, p.1007-1017, 2015 
à metade do valor de VI da espécie nativa de maior valor de importância.

Cada um dos oito blocos amostrados foi classificado em grupos sucessionais, baseado nos critérios da Resolução CONAMA 004/94 (CONAMA, 1994). A diferença entre esses grupos foi testada pela análise de variância multivariada permutacional (PERMANOVA) $(p<0,05)$. A organização florístico-estrutural da comunidade foi verificada pela Non metric Multidimensional Scaling (NMDS), que permitiu inferências sobre a similaridade entre as parcelas e a inserção de $H$. dulcis nos diferentes estágios sucessionais. A adequabilidade da ordenação foi verificada pelo valor de stress. Foi realizada a Análise de Espécies Indicadoras para detectar espécies com forte associação com cada grupo formado a partir da classificação sucessional. Essas análises foram realizadas no programa R (R DEVELOPMENT CORE TEAM, 2014), junto com o pacote Vegan (OKSANEN et al., 2014) para PERMANOVA e NMDS e labdsv (ROBERTS, 2014), para Análise de Espécies Indicadoras.

As parcelas foram classificadas como contaminadas e não contaminadas, de acordo com a presença ou ausência de $H$. dulcis. Os padrões de diversidade nos diferentes setores (contaminado e não contaminado) foram analisados pelos valores de riqueza, índices de diversidade de Shannon (H') e equabilidade de Pielou (J). A riqueza foi comparada pela técnica de rarefação $(\mathrm{n}=425$ indivíduos, valor do setor de menor densidade) e a diversidade pelo teste $t$, de Hutcheson (ZAR, 1996). A estruturação da comunidade foi avaliada pelo padrão de coexistência das espécies, utilizando o índice $c$-score, proposto por Stone e Roberts (1990), determinado com o programa EcoSim 7.0 (GOTELLI; ENTSMINGER, 2004). Assim, os valores de $c$-score observados para cada um dos setores relativos à presença ou ausência de invasão por $H$. dulcis foram comparados com os valores de $c$-score determinados a partir de um modelo nulo, produzido com 5.000 iterações; e do algoritmo de swap sequencial do EcoSim 7.0. Valores de $c$-score significativamente maiores do que os simulados pelo modelo nulo indicam uma comunidade estruturada, com predominância de segregação das espécies nas parcelas; valores de $c$-score abaixo dos valores simulados indicam uma comunidade estruturada, com predominância de coexistência das espécies; valores de $c$-score entre os valores simulados evidenciam uma comunidade sem estruturação, de padrão aleatório
(GOTELLI; ENTSMINGER, 2004). Os valores de -score dos setores contaminados e não contaminados foram comparados por meio do módulo Guild structure do mesmo programa $(\mathrm{p} \leq 0,1)$, em que é verificada a variância do índice, como descrito por Sanders et al. (2003).

\section{RESULTADOS}

Foram amostrados 924 indivíduos, pertencentes a 66 espécies e 30 famílias (Tabela 1). Fabaceae foi a família de maior riqueza (11 espécies). Foram amostradas três espécies exóticas: H. dulcis (33 indivíduos) e duas espécies de Citrus, que totalizaram 12 indivíduos. O índice de Shannon foi de 3,45 e a equabilidade de Pielou, de 0,82 . A curva de acumulação de espécies (Figura 2) tendeu à estabilidade $\mathrm{e}$, com a inclusão da última parcela, que representou $2,08 \%$ da área amostral, houve aumento de apenas $0,29 \%$ em espécies.

A espécie com maior Valor de Importância (VI) foi Ocotea puberula (Rich.) Nees (10,79\%), seguida por Nectandra megapotamica (Spreng.) Mez (10,73\%). H. dulcis foi a sétima mais importante $(4,05 \%)$, sendo o Índice de Invasão Biológica (IIB) de 0,47.

A classificação sucessional indicou a existência de diferenças florístico-estruturais entre os blocos $(p=0,001)$, confirmando a elevada heterogeneidade observada a campo. Os trechos amostrados pelos blocos 1, 2 e 3 indicaram um estágio sucessional intermediário, e os trechos amostrados pelos blocos 4, 5, 6, 7 e 8 indicaram um estágio sucessional avançado.

O valor de stress da NMDS foi de 17,84\%. Nela, foi possível observar a variação florístico-estrutural, com a distribuição das parcelas em diferentes estágios sucessionais, desde parcelas em trechos de floresta em estágio intermediário (parcelas ordenadas do lado esquerdo do gráfico, pertencentes aos blocos 1, 2 e 3$)$ até parcelas em trechos de floresta em estágios mais avançados de sucessão (parcelas do lado direito do gráfico, pertencentes aos blocos 4 a 8) (Figura 3). Do lado esquerdo do gráfico, foi observado Schinus terebinthifolius Raddi e Solanum mauritianum Scop., espécies pioneiras ou de início de estágio sucessional, e, do lado direito, Trichilia clausseni C.DC., espécie de maior tolerância à sombra, confirmando o gradiente sucessional encontrado na área. H. dulcis apresentou maior associação com o estágio intermediário de sucessão, agrupando-se as espécies do lado esquerdo do gráfico. 
Invasão biológica por Hovenia dulcis Thunb. em ...

Tabela 1 - Espécies amostradas, seguidas pelo número de indivíduos (N), Valor de Importância (VI, em \%) e grupo de estágio sucessional (ES) em que foi classificada, segundo a Análise de Espécies Indicadoras, em uma área de transição entre Floresta Estacional Decidual e Floresta Ombrófila Mista em Piratuba, SC.

Table 1 - Sampled species, followed by the number of individuals ( N), Importance Value (IV, in\%) and successional stage group (ES) according to the Indicator Species Analysis, in a transition area between Deciduous Forest and Araucaria Forest in the municipality of Piratuba, SC.

\begin{tabular}{|c|c|c|c|c|}
\hline Famílias & Espécies & $\mathrm{N}$ & VI & ES \\
\hline \multirow[t]{2}{*}{ Anacardiaceae } & Lithrea brasiliensis Marchand & 10 & 1,06 & - \\
\hline & Schinus terebinthifolius Raddi & 84 & 5,22 & $M(0,001)$ \\
\hline \multirow[t]{2}{*}{ Annonaceae } & Annona cacans Warm. & 2 & 0,25 & - \\
\hline & Annona sylvatica A.St.-Hil. & 6 & 0,58 & - \\
\hline Apocynaceae & Rauvolfia sellowii Müll. Arg. & 1 & 0,13 & - \\
\hline Aquifoliaceae & Ilex paraguariensis A.St.-Hil. & 2 & 0,24 & - \\
\hline Araucariaceae & Araucaria angustifolia (Bertol.) Kuntze & 1 & 0,12 & - \\
\hline Arecaceae & Syagrus romanzoffiana (Cham.) Glassman & 8 & 1,40 & $\mathrm{~A}(0,035)$ \\
\hline \multirow[t]{2}{*}{ Boraginaceae } & Cordia americana (L.) Gottschling \& J.S. Mill. & 18 & 2,08 & $M(0,001)$ \\
\hline & Cordia trichotoma (Vell.) Arrab. ex Steud. & 4 & 0,53 & - \\
\hline Cannabaceae & Celtis iguanaea (Jacq.) Sarg. & 8 & 0,86 & - \\
\hline Cardiopteridaceae & Citronella gongonha (Mart.) R.A. Howard & 2 & 0,25 & - \\
\hline Caricaceae & Vasconcellea quercifolia A.St.-Hil. & 5 & 0,47 & - \\
\hline \multirow[t]{5}{*}{ Euphorbiaceae } & Actinostemon concolor (Spreng.) Müll. Arg. & 15 & 1,43 & - \\
\hline & Alchornea triplinervia (Spreng.) Müll. Arg. & 2 & 0,22 & - \\
\hline & Gymnanthes klotzschiana Müll. Arg. & 9 & 0,94 & $\mathrm{M}(0,002)$ \\
\hline & Manihot grahamii Hook. & 1 & 0,11 & - \\
\hline & Sapium glandulosum (L.) Morong & 17 & 2,20 & $\mathrm{M}(0,001)$ \\
\hline \multirow[t]{12}{*}{ Fabaceae } & Albizia edwallii (Hoehne) Barneby \& J.W. Grimes & 12 & 1,53 & - \\
\hline & Apuleia leiocarpa (Vogel) J.F. Macbr. & 12 & 1,06 & - \\
\hline & Ateleia glazioveana Baill. & 1 & 0,11 & - \\
\hline & Bauhinia forficata Link & 10 & 0,94 & - \\
\hline & Dalbergia frutescens (Vell.) Britton & 10 & 0,73 & $\mathrm{M}(0,018)$ \\
\hline & Erythrina falcata Benth. & 1 & 0,12 & - \\
\hline & Machaerium stipitatum Vogel & 23 & 3,04 & - \\
\hline & Mimosa bimucronata (DC.) Kuntze & 5 & 0,38 & - \\
\hline & Muellera campestres (Mart. ex Benth.) & & & \\
\hline & M.J. Silva \& A.M.G. Azevedo & 64 & 5,82 & - \\
\hline & Myrocarpus frondosus Allemão & 8 & 0,92 & - \\
\hline & Parapiptadenia rigida (Benth.) Brenan & 3 & 0,50 & - \\
\hline \multirow[t]{4}{*}{ Lauraceae } & Nectandra lanceolata Nees & 1 & 0,18 & - \\
\hline & Nectandra megapotamica (Spreng.) Mez & 72 & 10,73 & $\mathrm{~A}(0,001)$ \\
\hline & Ocotea odorifera (Vell.) Rohwer & 14 & 1,76 & - \\
\hline & Ocotea puberula (Rich.) Nees & 109 & 10,79 & - \\
\hline Loganiaceae & Strychnos brasiliensis Mart. & 2 & 0,25 & - \\
\hline Malvaceae & Luehea divaricata Mart. \& Zucc. & 58 & 7,09 & - \\
\hline \multirow[t]{3}{*}{ Meliaceae } & Cabralea canjerana (Vell.) Mart. & 5 & 0,70 & - \\
\hline & Cedrela fissilis Vell. & 14 & 2,66 & $\mathrm{~A}(0,013)$ \\
\hline & Trichilia claussenii C.DC. & 19 & 1,71 & - \\
\hline \multirow[t]{2}{*}{ Moraceae } & Ficus luschnathiana (Miq.) Miq. & 4 & 0,54 & - \\
\hline & Maclura tinctoria (L.) Don ex Steud. & 3 & 0,43 & - \\
\hline \multirow[t]{5}{*}{ Myrtaceae } & Calyptranthes tricona D. Legrand & 13 & 1,61 & $\mathrm{~A}(0,018)$ \\
\hline & Campomanesia guazumifolia (Cambess.) Berg. & 4 & 0,49 & - \\
\hline & Campomanesia xanthocarpa (Mart.) O. Berg & 3 & 0,63 & - \\
\hline & Eugenia uniflora $\mathrm{L}$. & 4 & 0,67 & - \\
\hline & Myrcia oblongata DC. & 30 & 3,40 & $\mathrm{~A}(0,002)$ \\
\hline Phytolaccaceae & Phytolacca dioica $\mathrm{L}$. & 3 & 0,53 & - \\
\hline
\end{tabular}


Tabela 1 - Cont.

Table 1 - Cont.

\begin{tabular}{|c|c|c|c|c|}
\hline Famílias & Espécies & $\mathrm{N}$ & VI & ES \\
\hline Primulaceae & Myrsine umbellata Mart. & 3 & 0,44 & - \\
\hline Rhamnaceae & Hovenia dulcis Thunb. & 33 & 4,05 & $\mathrm{M}(0,037)$ \\
\hline Rosaceae & Prunus myrtifolia (L.) Urb. & 12 & 1,61 & $\mathrm{M}(0,002)$ \\
\hline \multirow[t]{6}{*}{ Rutaceae } & Balfourodendron riedelianum (Engl.) Engl. & 1 & 0,12 & - \\
\hline & Citrus reticulata Blanco & 10 & 1,04 & $\mathrm{M}(0,006)$ \\
\hline & Citrus $x$ aurantium $\mathrm{L}$. & 2 & 0,26 & - \\
\hline & Pilocarpus pennatifolius Lem. & 11 & 1,17 & $\mathrm{~A}(0,042)$ \\
\hline & Zanthoxylum petiolare A.St.-Hil. \& Tul. & 3 & 0,28 & - \\
\hline & Zanthoxylum rhoifolium Lam. & 15 & 1,44 & - \\
\hline \multirow[t]{2}{*}{ Salicaceae } & Casearia decandra Jacq. & 3 & 0,38 & - \\
\hline & Casearia sylvestris $\mathrm{Sw}$. & 67 & 5,49 & $\mathrm{M}(0,001)$ \\
\hline \multirow[t]{4}{*}{ Sapindaceae } & Allophylus puberulus (Cambess.) Radlk. & 6 & 0,76 & - \\
\hline & Cupania vernalis Cambess. & 29 & 2,63 & - \\
\hline & Diatenopteryx sorbifolia Radlk. & 2 & 0,41 & - \\
\hline & Matayba elaeagnoides Radlk. & 7 & 0,63 & - \\
\hline Sapotaceae & Chrysophyllum gonocarpum (Mart. \& Eichler ex Miq.) Engl. & 2 & 0,25 & - \\
\hline Simaroubaceae & Picrasma crenata (Vell.) Engl. & 1 & 0,17 & - \\
\hline Solanaceae & Solanum mauritianum Scop. & 7 & 0,90 & - \\
\hline Styracaceae & Styrax leprosus Hook. \& Arn. & 6 & 0,33 & - \\
\hline Verbenaceae & Aloysia virgata (Ruiz \& Pav.) Juss. & 2 & 0,24 & - \\
\hline Totais & & 924 & 100,0 & - \\
\hline
\end{tabular}

$\mathrm{A}=$ estágio sucessional avançado e $\mathrm{M}=$ estágio sucessional médio. Valores entre parênteses indicam a significância da análise $(p=0,05)$.

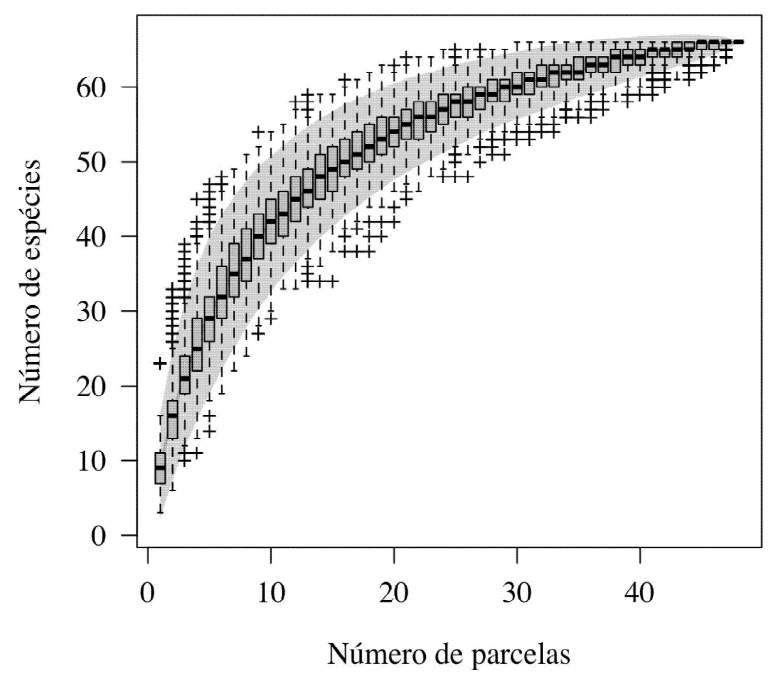

Figura 2 - Curva de acumulação das espécies amostradas em uma área de transição entre Floresta Estacional Decidual e Floresta Ombrófila Mista em Piratuba, SC.

Figure 2-Accumulation curve of the species sampled in a transition area between Deciduous Forest and Araucaria Forest in the municipality of Piratuba, SC.

Revista Árvore, Viçosa-MG, v.39, n.6, p.1007-1017, 2015

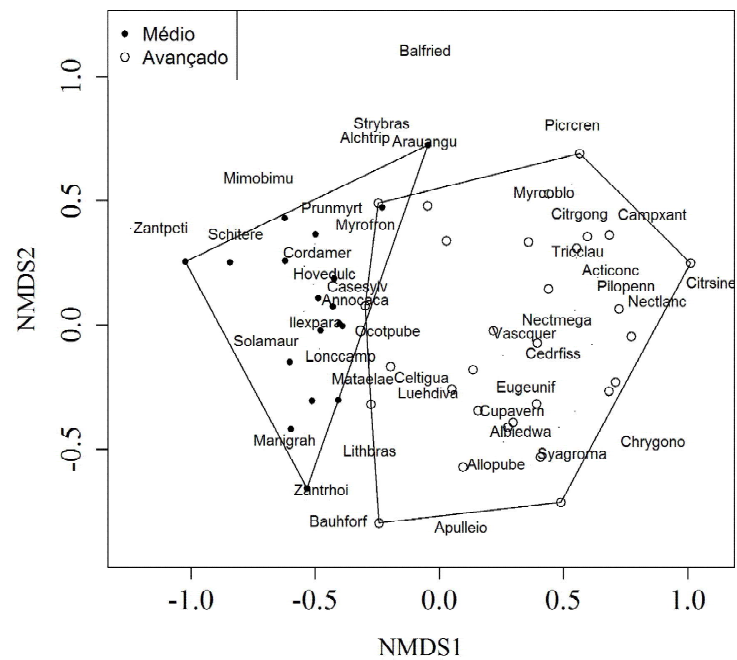

Figura 3 - Diagrama de ordenação das parcelas e espécies, produzido pela análise NMDS (escalonamento multidimensional não métrico), na área de transição entre Floresta Estacional Decidual e Floresta Ombrófila Mista em Piratuba, SC.

Figure 3 - Ordination Diagram of plots and species produced by NMDS (non-metric multidimensional scaling) analysis, in a transition area between Deciduous Forest and Araucaria Forest in the municipality of Piratuba, SC. 
A Análise de Espécies Indicadoras confirmou os padrões observados na NMDS, pois $H$. dulcis foi classificada como indicadora de estágio sucessional intermediário, assim como Schinus terebinthifolius, Cordia americana (L.) Gottschling \& J.S. Mill., Sapium glandulosum (L.) Morong, entre outras (Tabela 1). No entanto, como indicadora de estágio sucessional avançado, foi possível observar outra Lauraceae, Nectandra megapotamica (Spreng.) Mez e algumas Myrtaceae, como Calyptranthes tricona D. Legrand.

Ao analisar as parcelas de acordo com a presença ou ausência de $H$. dulcis (contaminada e não contaminada), observou-se que, apesar de não haver diferença significativa da riqueza determinada pela rarefação, a área contaminada apresentou menor diversidade e maior dominância ecológica (Tabela 2). O c-score indicou, para a área não contaminada por H. dulcis, um padrão de segregação das espécies, o que demonstra que a distribuição delas nessa área ocorre na forma de mosaico. Para a área contaminada, o padrão foi aleatório, indicando comunidade não estruturada. Observou-se, também, diferença significativa no índice c-score entre a área contaminada e não contaminada (variância observada maior do que a estimada pelo modelo nulo, $p=0,057$ ), com a existência de maior estruturação, a partir da maior segregação das espécies, no setor não contaminado.

\section{DISCUSSÃO}

A natureza ecotonal da floresta estudada é confirmada com a presença de espécies características de FED e FOM: algumas espécies amostradas, como Albizia edwallii (Hoehne) Barneby \& J.W., Grimes e Apuleia leiocarpa (Vogel) J.F. Macbr., são indicadoras do padrão estacional da área, no entanto espécies características da FOM, como Araucaria angustifolia (Bertol.) Kuntze, também foram encontradas. Porém, a família de maior número de espécies - Fabaceae confirma uma tendência mais estacional, já que, em geral, essa é a família de maior riqueza em florestas estacionais de Santa Catarina (GASPER et al., 2013a). Já em áreas de FOM essa família geralmente é substituída por Myrtaceae e Asteraceae como as de maior número de espécies (HIGUCHI et al., 2012; SILVA et al., 2012; GASPER et al., 2013b).

Apesar da presença de três espécies exóticas, o valor de diversidade encontrado $\left(H^{\prime}=3,45\right)$ foi elevado e similar ao encontrado por Longhi et al. (2000) (H' $=3,21)$ em um fragmento da FED. Portanto, a presença de espécies exóticas parece não ter influenciado, ainda, a diversidade. $\mathrm{O}$ alto valor de equabilidade de Pielou $(\mathrm{J}=0,82)$ indicou baixa dominância ecológica. Considerando que a floresta passou por distúrbios recentes, não eram esperados valores altos das estimativas de H' e J. No entanto, esse pode ser indicativo, no caso da diversidade, da elevada heterogeneidade florístico-estrutural observada na área, com trechos da floresta com diferentes históricos de perturbação, o que pode elevar valores de H'. A suficiência amostral foi atingida, pois, segundo Kersten e Galvão (2011), atinge-se a suficiência quando a linha tende à estabilidade, e o aumento do esforço amostral não altera significativamente o número de espécies observadas, sendo sugerido que aumento de $10 \%$ na área amostral resulta aumento inferior a 5\% no número de espécies. A elevada importância de espécies de canelas, como O. puberula, é comum em florestas deciduais, como a estudada por Longhi et al. (2000) em Santa Maria, $\mathrm{RS}$, onde foi encontrado, nessa espécie, VI de 16,51\%. Em relação à espécie exótica de maior VI, H. dulcis,

Tabela 2 - Riqueza (S), diversidade ( $\left.\mathrm{H}^{\prime}\right)$, equabilidade (J) e índices c-score calculados para as áreas não contaminadas (NC) e contaminadas (C) por Hovenia dulcis em uma área de transição entre Floresta Estacional Decidual e Floresta Ombrófila Mista em Piratuba, SC.

Table 2-Richness $(S)$, diversity ( $\left.H^{\prime}\right)$, evenness $(J)$ and c-score indices calculated for not contaminated areas $(N C)$ and contaminated (C) by Hovenia dulcis in a transition area between Deciduous Forest and Araucaria Forest in Piratuba, SC.

\begin{tabular}{|c|c|c|c|c|c|c|c|c|c|}
\hline & \multirow{2}{*}{$\mathrm{S}$} & \multirow{2}{*}{$\mathrm{H}^{\prime}$} & \multirow{2}{*}{$\mathbf{J}$} & \multirow{2}{*}{$p\left(\mathrm{H}^{\prime}\right)$} & \multicolumn{2}{|c|}{ Matriz Randomizada } & \multirow{2}{*}{ Obs } & \multicolumn{2}{|c|}{$p(\mathrm{c})$} \\
\hline & & & & & Min. & Max & & Obs.<esp. & Obs.>esp. \\
\hline $\mathrm{NC}$ & $55 \pm 1$ & 3,36 & 0,83 & 0,025 & 9,65 & 10,06 & 10,10 & 1,000 & 0,000 \\
\hline $\mathrm{C}$ & 54 & 3,17 & 0,79 & & 3,89 & 4,43 & 4,33 & 0,994 & 0,006 \\
\hline
\end{tabular}

Min. e Max. representam os valores mínimos e máximos estimados para matrizes randomizadas 5.000 vezes; $p$ (H') $=$ significância do teste t de Hutcheson para a comparação da diversidade entre área contaminada e não contaminada; Obs = valores de $c$-score observados; $p(\mathrm{c})=$ probabilidade de valores observados (obs.) serem maiores ou menores do que o esperado (esp.). 
sua importância elevada na comunidade estudada reforça a ideia de seu caráter invasor em remanescentes de áreas de ecótono entre FED e FOM na Região Sul do Brasil, como já observado por Meyer et al. (2012) em diferentes fitofisionomias. No entanto, o IIB encontrado indica que o grupo de espécies invasoras possui importância relativa inferior à metade do valor do VI da espécie nativa de maior importância, ou seja, o valor não foi muito elevado. Os IIBs encontrados por Guidini et al. (2014), por exemplo, para duas comunidades arbóreas de FOM com a invasão de Citrus $x$ limon (L.) Osbeck, Ligustrum lucidum W.T. Aiton, Pinus elliottii Engelm. e Pinus taeda L., foram de 0,61 e 0,05. Vale ressaltar que o processo de contaminação biológica, normalmente, é caracterizado por uma fase inicial, conhecida como lag, em que a espécie pode ocorrer com baixa frequência, e o aumento populacional é inexistente ou ocorre em baixa intensidade, sucedida por uma fase com forte aumento populacional (AIKIO et al., 2010). Dessa forma, o IIB relativamente baixo sugere que as espécies nativas, ainda, apresentam forte dominância ecológica na área. Assim, infere-se que as espécies invasoras estejam em uma fase de estabelecimento na área, fase lag, podendo aumentar sua importância ou, em razão da competição interespecífica, as espécies nativas dominantes estejam impedindo maior invasão biológica.

A classificação dos blocos amostrados em estágios sucessionais médio e avançado confirmou o padrão observado a campo, pois os blocos 1, 2 e 3 eram, de fato, áreas compostas por indivíduos arbóreos adultos provenientes da regeneração natural recente de áreas de pastagem ou lavoura. Porém, o bloco 4, que também era uma área de pastagem ou de lavoura, foi classificado como de estágio sucessional avançado. Essas diferenças na classificação para áreas com um mesmo histórico de perturbação podem ser explicadas pela diferença de resiliência de cada área (e.g. fonte de propágulo, condições edáficas).

O valor de stress da NMDS indica que os diagramas de ordenação são adequados para interpretação, pois, considerando que esse valor varia de 0 a $100 \%$, o baixo valor encontrado demonstra um bom ajuste linear entre as distâncias euclidianas das projeções das parcelas na ordenação e a similaridade florístico-estrutural entre elas (BORCARD et al., 2011). As espécies características dos estágios de sucessão observados na NMDS confirmam os grupos sucessionais já observados por outros autores, por exemplo os de Schinus terebinthifolius e Solanum mauritianum, que também foram espécies comuns em áreas alteradas de FED Aluvial em Santa Maria, RS, estudadas por Bianchin e Bellé (2013).

H. dulcis, que se agrupou junto com as espécies mais iniciais e foi classificada como preferencial de estágio sucessional intermediário, foi observada no Inventário Florístico Florestal de Santa Catarina (MEYER et al., 2012) também de forma associada a áreas perturbadas. Assim, o resultado encontrado neste estudo e o da literatura sugerem que florestas iniciais ou impactadas são mais sensíveis à invasão por essa espécie. Já espécies de Lauraceae, mais comuns nos trechos de sucessão mais avançados, são frequentes em áreas mais conservadas de FED, conforme verificado por Vaccaro et al. (1999) em Santa Tereza, RS.

A menor diversidade e a maior dominância nas áreas contaminadas por $H$. dulcis podem ser explicadas pela associação da espécie com o setor em estágio mais inicial de sucessão, uma vez que nessa etapa a diversidade tende a ser menor (RODRIGUES et al., 2004; SIMINSKI et al., 2004), em razão da maior dominância de algumas espécies. Levine (2000) demonstrou que a diversidade residente pode aumentar a resistência à invasão biológica, principalmente em pequenas escalas espaciais, em que interações entre indivíduos vizinhos ocorrem.

$\mathrm{Na}$ área não contaminada por $H$. dulcis, a maior segregação das espécies indicou que a organização da comunidade no setor sem contaminação apresenta sinal mais forte do processo de competição interespecífica, com maior substituição das espécies nas parcelas. Segundo Gotelli e McCabe (2002), essa forma de distribuição é esperada em decorrência da relação de interações competitivas entre espécies em diferentes condições ambientais. Assim, pode-se inferir que $H$. dulcis ocupa, preferencialmente, sítios onde interações de competição entre espécie ocorrem com menor intensidade, em que a diversidade é menor e, conforme observado na NMDS, em sítios mais iniciais de sucessão.

\section{CONCLUSÃO}

Os resultados confirmaram a área estudada como ecotonal entre FED e FOM e H. dulcis como importante espécie invasora em florestas da região do Alto Uruguai. Destaca-se que o processo de invasão não ocorreu 
de forma espacialmente homogênea, estando associado ao contexto sucessional e ecológico da área, uma vez que a espécie ocorreu preferencialmente em locais em estágio mais inicial de sucessão, com menor diversidade e ausência de estruturação da comunidade, referente à segregação das espécies nas parcelas. Assim, infere-se que eventos que promovam o retorno de áreas florestais a estágios sucessionais mais iniciais, como distúrbios, favorecem o processo de invasão pela espécie.

\section{AGRADECIMENTOS}

À FAPESC, pela concessão de bolsa à primeira autora; e ao CNPq, pela concessão de bolsa de produtividade ao segundo e ao terceiro autor.

\section{REFERÊNCIAS}

AIKIO, S.; DUNCAN, R.P.; HULME, P.E. Lag phases in alien plant invasions: separating the facts from the artefacts. Oikos, v.119, n.2, p. $370-378,2010$

APG III. An update of the Angiosperm Phylogeny Group classification for the orders and families of flowering plants: APG III.

Botanical Journal of the Linnean Society, v.161, n.2, p.105-121, 2009.

BIANCHIN, J.E.; BELLÉ, P.A. Fitossociologia e estrutura de um fragmento de Floresta Estacional Decidual Aluvial em Santa Maria RS. Revista Agro@mbiente On-line, v.7, n.3, p.322-330, 2013.

BORCARD, D.; GILLET, F.; LEGENDRE, P. Numerical ecology with R. Nova York: Springer, 2011.306p.

CONAMA. Resolução do Conama n. 04, de 4 de maio de 1994. Define vegetação primária e secundária nos estágios inicial, médio e avançado de regeneração da Mata Atlântica, a fim de orientar os procedimentos de licenciamento de atividades florestais em Santa Catarina. Diário Oficial da União, n. 114, p. 8877-8878, 17 jun. de 1994.

DAVIS, M.A.; GRIME, J.P.; THOMPSON, K. Fluctuating resources in plant communities: a general theory of invasibility. Journal of Ecology, v. 88, n.3, p.528-534, 2000.
DAVIS, M.A.; THOMPSON, K.; GRIME, J.P. Invasibility: the local mechanism driving community assembly and species diversity. Ecography, v.28, n.5, p.696-704, 2005.

GASPER, A. L.; UHLMANN, A.; SEVEGNANI, L.; VIBRANS, A. C.; LINGNER, D. V.; RIGON-JÚNIOR, M. J.; VERDI, M., STIVAL-SANTOS, A.; DREVECK, S.; SOBRAL, M. Inventário florístico florestal de Santa Catarina: espécies da Floresta Estacional Decidual. Rodriguésia, v.64, n.3, p.427-443, 2013a.

GASPER, A. L.; SEVEGNANI, L.; VIBRANS, A. C.; SOBRAL, M.; UHLMANN, A.; LINGNER, D. V.; RIGON-JÚNIOR, M. J.; VERDI, M., STIVALSANTOS, A.; DREVECK, S.; KORTE, A. Inventário florístico florestal de Santa Catarina: espécies da Floresta Ombrófila Mista. Rodriguésia, v.64, n.2, p.201-210, 2013 b.

GOTELLI, N.J.; MCCABE, D.J. Species cooccurrence: a meta-analysis of JM Diamond's assembly rules model. Ecology, v.83, n.8, p.20912096, 2002.

GOTELLI, N.J.; ENTSMINGER, G.L. EcoSim: Null models software for ecology. Version 7.Acquired Intelligence Inc. \& Kesey-Bear. Jericho, VT 05465. 2004. Disponível em: http://garyentsminger.com/ ecosim/index.htm. Acesso em: 2 nov. de 2014.

GUIDINI, A. L., SILVA, A. C, HIGUCHI, P., DALLA ROSA, A, SPIAZZI, F. R., NEGRINI, M., FERREIRA, T. S., SALAMI, B., MARCON, A. K.; BUZZI JUNIOR, F. Invasão por espécies arbóreas exóticas em remanescentes florestais no Planalto Sul Catarinense. Revista Árvore, v.38, n.3, p.469-478, 2014.

HIGUCHI, P.; SILVA, A.C.; FERREIRA, T. S.; SOUZA, S. T.; GOMES, J. P.; SILVA, K. M.; SANTOS, K. F.; LINKE, C.; PAULINO, P. S. Influência de variáveis ambientais sobre o padrão estrutural e florístico do componente arbóreo em um fragmento de Floresta Ombrófila Mista Montana, em Lages, SC. Ciência Florestal, v.22, n.1, p.79-90, 2012.

INSTITUTO BRASILEIRO DE GEOGRAFIA E ESTATÍSTICA - IBGE. Manual técnico da vegetação brasileira. Rio de Janeiro: 2012. 271p.

Revista Árvore, Viçosa-MG, v.39, n.6, p.1007-1017, 2015 
KERSTEN, R.A.; GALVÃO, F. Suficiência amostral em inventários florísticos e fitossociológicos. In: FELFILI, J.M. et al. (Eds.). Fitossociologia no Brasil: métodos e estudos de casos. Viçosa, MG: UFV, 2011.p.153-176.

LEVINE, J.M. Species diversity and biological invasions: relating local process to community pattern. Science, v.288, n.5467, p.852-854, 2000 .

LONGHI, S. J.; ARAUJO, M. M.; KELLING, M. B.; HOPPE, J. M.; MÜLLER, I.; BORSOI, G. A. Aspectos fitossociológicos de fragmento de floresta estacional decidual, Santa Maria, RS. Ciência Florestal, v. 10, n.2, p.59-74, 2000.

LORENZI, H.; SOUZA, H. M.; BACHER, L. B.; TORRES, M. A. V. Árvores exóticas no Brasil: madeireiras, ornamentais e aromáticas. Nova Odessa: Instituto Plantarum, 2003. 368p.

MEYER, L.; VIBRANS, A.C.; GASPER, A.L.; LINGNER, D.V.; SAMPAIO, D. K. Espécies exóticas encontradas nas florestas de Santa Catarina. In: VIBRANS, A.C. et al. (Eds.). Diversidade e conservação dos remanescentes florestais. Blumenau: Edifurb, 2012. v.1.p.236-265.

MUlleR-DOMBOIS, D.; ELLENBERG, H. Aims and of vegetation ecology. New York: John Wiley \& Sons, 1974. 547p.

OKSANEN, J.; BLANCHET, F. G.; KINDT, R.; LEGENDRE, P. MINCHIN, P. R.; O'HARA, R. B.; SIMPSON, G. L.; SOLYMOS, P.; STEVENS, M. H. H.; WAGNER, H. Vegan: Community ecology package. Disponível em: $<$ http://cran.rproject.org/> Acesso em: 11 set. de 2014.

PERRINGS, C.; WILLIAMSON, M.; BARBIER, E. B.; DELFINO, D.; DALMAZZONE, S.; SHOGREN, J.; SIMMONS, P.; WATKINSON, A. Biological invasion risks and the public good: an economic perspective. Conservation Ecology, v.6, n.1, p. 1, 2002.

PIMENTEL, D.; MCNAIR, S.; JANECKA, J.; WIGHTMAN, J.; SIMMONDS, C.; O'CONNEL, C.; WONG, E.; RUSSEL, L.; ZERN, J.; AQUINO, T.; TSOMONDO, T. Economic and environmental threats of alien plant, animal, and microbe invasions. Agriculture, Ecosystems \& Environment, v.84, n.1, p.1-20, 2001.

R DEVELOPMENT CORE TEAM. R: A language and environment for statistical computing. Disponível em: http://www.r-project.org/ Acesso em: 11 set. de 2014 .

ROBERTS, D. W. Labdsv: Ordination and multivariate analysis for ecology. R package version 1.6-1. Disponível em: <http:// CRAN.Rproject.org/package=labdsv. Acesso em: 11 set. de 2014 .

RODRIGUES, R.R.; MARTINS, S.V.; BARROS, L.C. Tropical rain forest regeneration in an area degraded by mining in Mato Grosso State, Brazil. Forest Ecology and Management, v.190, n.2, p.323-333, 2004.

SANDERS, N.J.; GOTELLI, N. J.; HELLER, N. E.; GORDON, D.M. Community disassembly by an invasive species. Proceedings of the National Academy of Sciences, v.100, n.5, p.2474-2477, 2003.

SILVA, A. C.; HIGUCHI, P.; AGUIAR, M. D.; NEGRINI, M.; FERT NETO, J.; HESS, A. F. Relações florísticas e fitossociologia de uma Floresta Ombrófila Mista Montana Secundária em Lages, Santa Catarina. Ciência Florestal, v.22, n.1, p.193-206, 2012.

SIMINSKI, A.; MANTOVANI, M.; REIS, M. S.; FANTINI, A. C. Sucessão florestal secundária no município de São Pedro de Alcântara, litoral de Santa Catarina: estrutura e diversidade. Ciência Florestal, v.14, n.1, p.21-33, 2004.

SOBRAL, M.; JARENKOW, J.A.; BRACK, P.; IRGANG, B.; LAROCCA, J.; RODRIGUES, R.S. Flora arbórea e arborescente do Rio Grande do Sul, Brasil. 2.ed. São Carlos: RiMa, 2013. 362p.

STONE, L.; ROBERTS, A. The checkerboard score and species distributions. Oecologia, v.85, n.1, p.74-79, 1990.

VACCARO, S.; LONGHI, S.J.; BRENA, D.A. Aspectos da composição florística e categorias sucessionais do estrato arbóreo de três subseres 
de uma Floresta Estacional Decidual, no município de Santa Tereza - RS. Ciência Florestal, v.9, n.1, p.1-18, 1999.

WANDSCHEER, A. C. D.; BORELLA, J.; BONATTI, L. C.; PASTORINI, L. H. Allelopathic activity of Hovenia dulcis Thunb. (Ramnaceae) leaves and pseudofruits on the germination of Lactuca sativa (Asteraceae). Acta Botanica
Brasilica, v.25, n.1, p.25-30, 2011.

ZAR, J.H. Biostatistical analysis. 3.ed. New Jersey: Prentice Hall, 1996. 662p.

ZILLER, S.R.; ZALBA, S. Propostas de ação para prevenção e controle de espécies exóticas invasoras. Natureza e Conservação, v. 5 , n. 2, p.8-15, 2007. 
